\title{
CREB Activation Mediates Plasticity in Cultured Hippocampal Neurons
}

\author{
Menahem Segal $^{1 \dagger}$ and Diane D. Murphy ${ }^{2}$ \\ ${ }^{1}$ Department of Neurobiology, The Weizmann Institute of Science, Rehovot 76100, Israel; \\ ${ }^{2}$ Laboratory of Neurobiology, NINDS, NIH, Bethesda, MD 20892, USA
}

\begin{abstract}
Activation of cyclic AMP dependent kinase is believed to mediate slow onset, long-term potentiation (LTP) in central neurons. CyclicAMP activates a cascade of molecular events leading to phosphorylation of the nuclear cAMP responsive element binding protein (pCREB). Whereas a variety of stimuli lead to activation of CREB, the molecular processes downstream of CREB, which may be relevant to neuronal plasticity, are yet largely unknown. We have recently found that following exposure to estradiol, pCREB mediates the large increase in dendritic spine density in cultured rat hippocampal neurons. We now extend these observations to include other stimuli, such as bicuculline, that cause the formation of new dendritic spines. Such stimuli share with estradiol the same mechanism of action in that both require activity-dependent CREB phosphorylation. Our observations suggest that CREB phosphorylation is a necessary, but perhaps not sufficient, step in the process leading to the generation of new dendritic spines and perhaps to functional plasticity as well.
\end{abstract}

\section{INTRODUCTION}

The cellular mechanisms underlying long-term synaptic plasticity have begun to emerge in a recent

\footnotetext{
${ }^{\dagger}$ Corresponding author: M. Segal

Tel: +972 89342 553, Fax: +972 89344140

e-mail: jnsegal@weizmann.weizmann.ac.il
}

series of studies linking plasticity-producing stimuli to the phosphorylation of the $c A M P$ responsive element binding protein (pCREB) (Nguyen \& Kandel, 1996; Deisseroff et al., 1996; Impey et al., 1996). The cyclic AMP-dependent activation of protein kinase $A$ (PKA) has been shown to be critical for maintaining the late phase of long-term potentiation (LTP) (Nguyen \& Kandel, 1996, Impey et al., 1996), and of extensive synaptic stimulation, which yields LTP in cultured neurons and leads to the calcium-dependent phosphorylation of CREB (Deisseroth et al., 1996). Behaviorally, pCREB is required for maintaining long-term, but not shortterm, memory (Lamprecht et al., 1997). Central to the study of long-term neuronal plasticity are dendritic spines, which are the primary targets of excitatory synaptic inputs and have been intimately associated with the long-term morphological modifications that are seen during LTP and behavioral plasticity (Chang \& Greenough, 1984; Fifkova \& Van Harreveld, 1977; Geinisman et al., 1988; Lee et al., 1980). Changes in spine morphology may affect the dendritic integration of synaptic potentials (Harris \& Kater, 1994). Despite an apparently pivotal role in synaptic plasticity, little is known about the molecular events linking CREB, neuronal plasticity, and the regulation of dendritic spine formation.

To study the molecular events linking these plasticity-related neuronal processes, we developed a tissue-culture system, in which dissociated rat hippocampal neurons are grown for several weeks. The neurons are then exposed to chemical stimulation and monitored for changes in dendritic morphology and spine density. In earlier studies, we found that cultured neurons develop dendritic spines during their 3 weeks in culture, and that these spines serve as the post-synaptic sites for connections 
among adjacent cells in the culture (Papa et al., 1995). We also found that exposure of the cultures to stimuli that enhance spontaneous activity (for example, blockade of inhibition by picrotoxin or bicuculline) cause the formation of dendritic spines (Papa \& Segal, 1996). More recently, we found that estradiol produces a two-fold increase in dendritic spine density in cultured hippocampal neurons (Murphy \& Segal, 1996), qualitatively similar to its effects in vivo (Woolley \& McEwen, 1994). As such, estradiol proved to be a convenient stimulus for analyzing the biological mechanisms regulating dendritic spine formation. For example, we found that the effect of estradiol is mediated by the activation of a serine/threonine kinase. Because estradiol activates cAMP-dependent PKA (Spaulding, 1993), and estradiol may affect CREB ( $\mathrm{Gu}$ et al., 1996), we explored the possible involvement of CREB in the action of estradiol on dendritic spine formation.

Estradiol produced a slow-onset, long-lasting increase in the immunoreactivity of nuclear pCREB and of the CREB-binding protein (CBP), which binds specifically to the PKA-phosphorylated form of CREB, and thus augments the ability of pCREB to activate the transcription of cAMP-responsive genes, as shown in Fig. 1 (see also Murphy \& Segal, 1997 and citations therein). From a baseline condition, in which about $15 \%$ of the cells were immunopositive for $\mathrm{CBP}$, the number of positive cells started to increase at $6 \mathrm{hr}$ after exposure to estradiol and reached a peak (80\%) at about $24 \mathrm{hr}$ after exposure. Likewise, whereas pCREB-staining was seen in only $13 \%$ of the cells at rest, the density of stained cells increased to $80 \%$ after $20 \mathrm{hr}$ of exposure to estradiol. The slow time course of the estradiol effect on pCREB and CBP is consistent with the slow time course of spine formation following exposure to estradiol (Murphy \& Segal, 1996) (also see below).

We found that the NMDA receptor plays a crucial role in the formation of spines by estradiol, as well as in the activation of CREB by estradiol, because these effects were blocked by the NMDA antagonist, 2-APV (Murphy \& Segal, 1996). NMDA-receptor activation is associated with neuronal plasticity because it allows a rise of intracellular calcium concentration $\left([\mathrm{Ca}]_{i}\right)$. The involvement of $[\mathrm{Ca}]_{i}$ in pCREB formation was established by using the cell-permeant calcium chelator BAPTA-AM (Deisseroth et al., 1996). Indeed, BAPTA-AM, which by itself caused a significant reduction in the mean CBP fluorescence intensity of the measured neurons, also blocked the response to estradiol (Murphy \& Segal, 1997). These observations are somewhat puzzling in that if estradiol acts on cytosolic estrogen receptors to activate CREB directly, where is the membraneNMDA link in this cascade? The further studies described below clarify this issue.

We also studied the effects of a specific antisense oligonucleotide to CREB to determine if the estradiol-induced CREB formation and subsequent increase in spines could be eliminated by a nonpharmacological, nuclear probe. Cells treated for $36 \mathrm{hr}$ with antisense in the presence of estradiol display significantly less CREB fluorescence in comparison with those treated with sense and ordinary media (Murphy \& Segal, 1997). After exposure to estradiol, the spine density measured in DiI-stained cells increases by $69 \%$ in the sensecontrol culture, but is not affected by the hormone in antisense treated cells.

The universality of the CREB mediation of spine formation was also tested following exposure of the cultures to the GABAergic-receptor antagonist, bicuculline. Bicuculline causes an increase in spine density (Papa \& Segal, 1996), which is significant as early as $6 \mathrm{hr}$ after onset of exposure and then peaks $24 \mathrm{hr}$ later. Bicuculline also causes a large increase in pCREB immunoreactivity within $0.5 \mathrm{hr}$ after exposure to the drug, reaching a peak at $4 \mathrm{hr}$ and then returning to baseline after $8 \mathrm{hr}$ (Fig. 1). Thus, both estradiol and bicuculline share an effect on CREB, but with a marked difference in the time course.

In our more recent studies, the GABAergic interneuron was implicated in the action of estradiol (Murphy et al., 1998). Estrogen receptors are localized exclusively on aspiny GABAergic interneurons, as seen in vivo (Weiland et al., 1995). This observation means that the effects of estradiol on spine formation in spiny pyramidal neurons, which lack an apparent estrogen receptor, must be indirect. We found that estradiol downregulates activity is blocked by tetrodotoxin, and also in cultures treated 

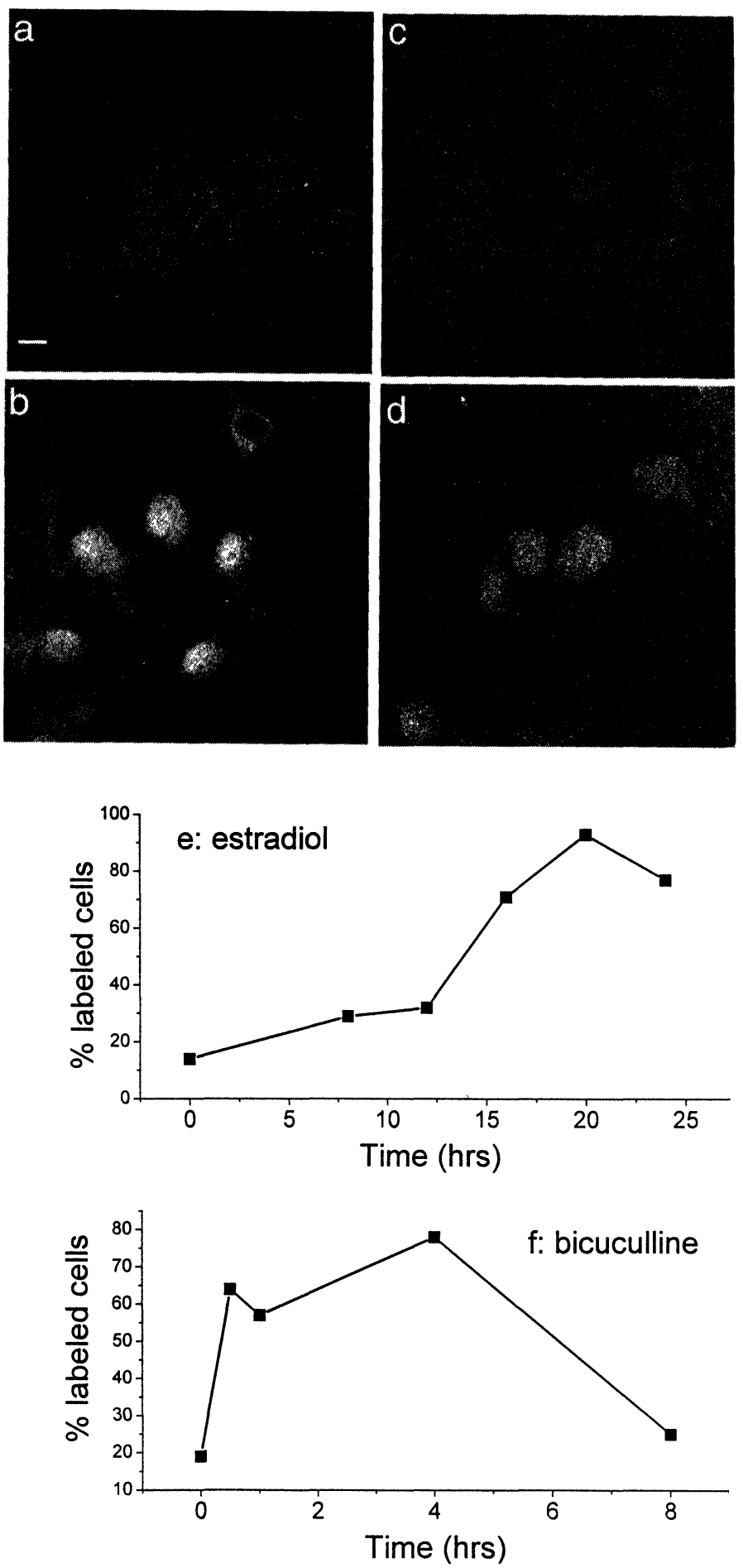

Fig. 1: A-D: Estradiol causes a rise in $\mathrm{PCREB}$ and $C B P$ immunoreactivity in nuclei of cultured hippocampal neurons, in a slow time dependent manner. Illustrations of cultured neurons stained for CBP and $\mathrm{pCREB}$ in control and following estradiol treatment $(0.1 \mu \mathrm{M}$, for $24 \mathrm{hrs}) \mathrm{A}$ and C, are controls, B and D are estradiol-treated. A \& B: CBP staining in neurons, C\&D: pCREB staining in neurons (modified from Murphy \& Segal, 1997). E: Time course of pCREB following exposure to estradiol. Percentage of cells with nuclear stain of pCREB is presented as a function of time after onset of exposure to the drug, as seen above (see Murphy \& Segal, 1997 for details). F: Time course of CREB phosphorylation following exposure of the culture to bicuculline $(50 \mu \mathrm{M})$. More cells are responding by an increase in pCREB after exposure to bicuculline; the effect is transient and returns to baseline within $8 \mathrm{hr}$ of exposure to the drug. For each time point between 90 and $125 \mathrm{~min}$, cells were scanned using NIH Image software, and the results were either quantified or thresholded and expressed as active cells over the total. 


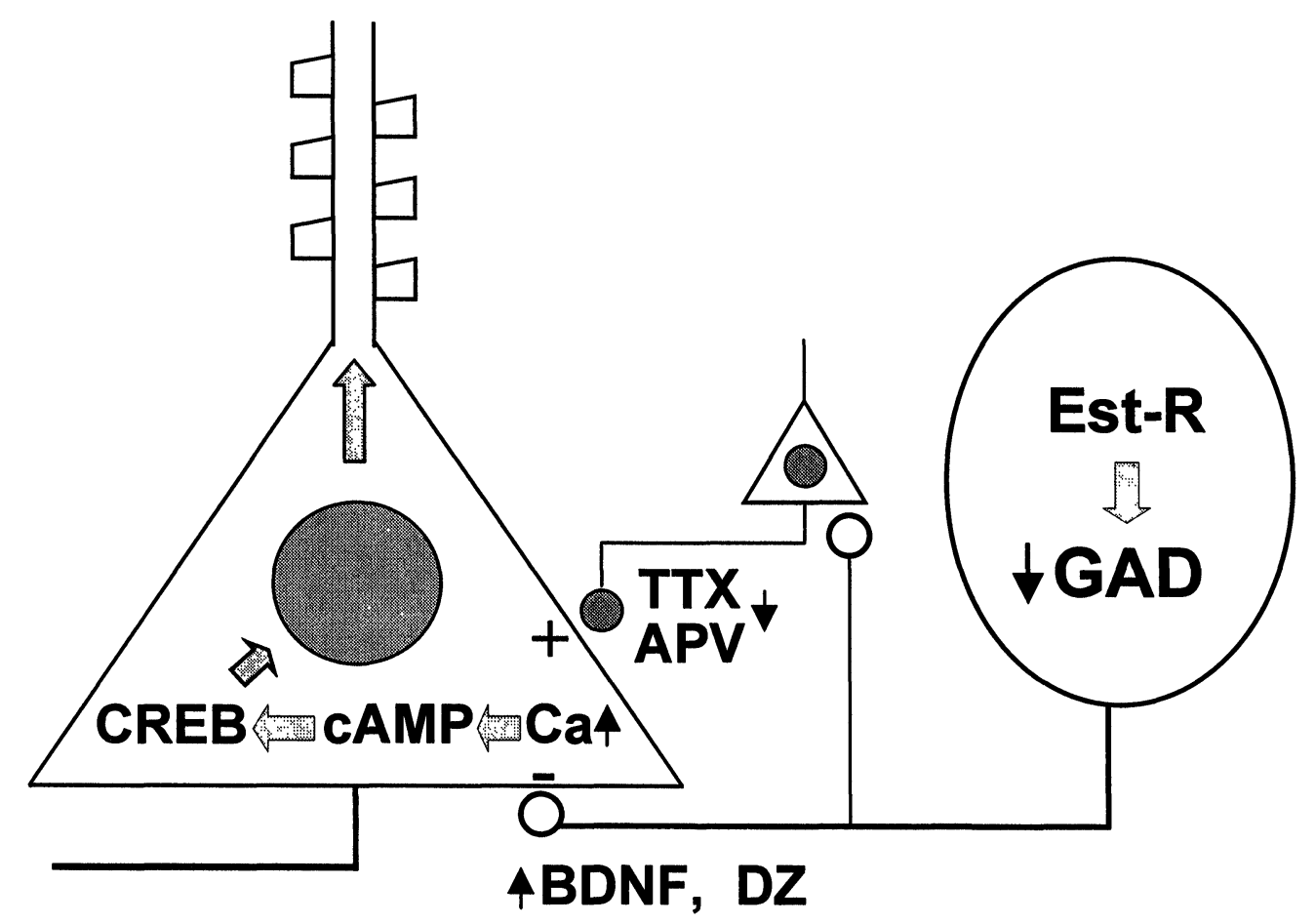

Fig. 2: Schematic diagram of the current hypothesis on the link between estradiol, calcium, CREB, and spine formation. Estrogen receptors in interneurons downregulate $\mathrm{GAD}$, which causes a reduced inhibition of pyramidal neurons (triangles). The increased excitation causes (a) an increased influx of calcium, (b) the formation of cAMP, pCREB, and dendritic spines. Adding either TTX or APV blocks the effect of estradiol by reducing excitation; BDNF and diazepam (DZ) block the effects of estradiol by enhancing inhibition.

with drugs that potentiate ongoing GABAtrgic inhibitory activity, for example diazepam (which prolongs the duration of the GABA channel open time). In the same series of experiments, we found that both TTX and diazepam eliminate the pCREB response to estradiol, indicating that the hormone causes its effect on spine formation by reducing GABA efficacy in the culture, leading to (a) increased excitatory network activity, (b) activation of NMDA-mediated calcium influx , and (c) CREB phosphorylation.

The time course of the effect of estradiol on pCREB and CBP (20-24 hr to reach the peak) is slower in comparison with other reported immediate early genes (Deisseroth et al., 1996) and the fast kinetics of $\mathrm{PCREB}$ activation seen with bicuculline. Such a slow time course is consistent with the time course of spine formation following exposure to estradiol, which peaks $48-72 \mathrm{hr}$ after the onset of exposure (Murphy \& Segal, 1996). This slow time course results from the estradiol-induced, gradual reduction in GAD activity, which precedes the change in spontaneous activity and enhances CREB, unlike the effect of bicuculline, where the change in activity is immediate. In either case, an increased action potential discharges, and excitatory synaptic potentials underlie the activation of CREB and the subsequent formation of novel dendritic spines.

Our results suggest the following:

- The activation of CREB is a common path for the production of new dendritic spines. An increase in action potential discharges causes an enhanced influx of calcium via NMDA dependent synaptic channels.

- The rise in intracellular calcium activates the cAMP cascade which, in turn, leads to CREB phosphorylation and the subsequent formation of new dendritic spines. 
Although attractively simple, this hypothesis raises several major questions. The biochemical route from the activated CREB to the morphological change in dendritic spines seen here is still uncharted. CREB can actually lead to the regulation of several different genes that are not necessarily related to the formation of either dendritic spines or memory. One recent illustration (Shieh et al., 1998, Tao et al., 1998) involves the CREB mediated upregulation of brain-derived neurotrophic factor (BDNF) which, in another recent study (VicarioAbejon et al., in press), reportedly does not affect spine formation. Thus, whether pCREB leads to the formation of dendritic spines probably depends on some other signaling molecule.

This probability was indeed the case in our recent studies, as we found that phosphorylation of CREB by a stable cAMP analogue (SP-cAMP) was not sufficient to mimic the production of spines that was seen with estradiol (Murphy \& Segal, 1997). The nature of this additional signal is still unknown, but we did find that SP-cAMP, when applied together with a depolarizing agent, does enhance the production of spines (unpublished observations).

An alternative possibility to be considered is that by itself, CREB activation can produce spines, but the extent of this action depends on the pattern of CREB activation rather than on the absolute level of activated CREB. For example, it was recently found that a cyclic elevation of intracellular calcium can enhance gene expression differentially in a way that is distinct from that produced by a continuous rise in [Ca $]_{i}$ (Dolmetsch et al., 1998). This finding can, in fact, explain our observations indicating that estradiol can actually produce a higher increase in spine density than that caused by a blockade of inhibition by bicuculline. In the latter case, the rise in $[\mathrm{Ca}]_{i}$ is fast and large, and can result in cell death. Likewise, a severe rise of $[\mathrm{Ca}]_{i}$, caused for example by the exposure of the cells to a calcium ionophore, causes cell death and not spine formation (unpublished observations). Thus, the pattern of the rise of $[\mathrm{Ca}]_{i}$, and its subsequent effect on CREB activation, its duration, and magnitude will determine the ability of CREB to cause the formation of dendritic spines.

Independent of its involvement in the formation of dendritic spines, CREB activation has been implicated in long-term memory storage (Lamprecht et al., 1997). Whether the formation of novel spines mediates this involvement of CREB in memory is not entirely clear, nor is whether CREB is also involved in the strengthening of existing spine or shaft synapses. The contrast between the assumed global roles of CREB in long-lasting memory storage and in CREB-mediated, estradiol-induced spine formation is puzzling because estradiol action on spine density in vivo is in constant flux, that is, the spines appear and disappear across the estrous cycle (Woolley et al. 1990).

Assuming that CREB is related to memory formation via the production of novel spines, then how is stimulus specificity retained through the CREB signal? How is an apparent specific 'memory' encoded through a general and nonselective nuclear CREB signaling molecule? Two molecular aspects to this issue are present.

1. The first aspect is that of the stimulation: Can we assume that CREB activation is linearly related to the amount and duration of the rise in $[\mathrm{Ca}]_{\mathrm{i}}$ and to the subsequent strength of the 'memory trace'?

2. Or, alternatively, is there something unique about a particular pattern of stimulation that produces memory more efficiently than other patterns, regardless of the CREB signaling molecule?

For example, one leading hypothesis in neuroplasticity is the Hebbian rule, that the simultaneous activation of pre- and post-synaptic neurons will strengthen the connection between them. Is the Hebbian condition simply a more efficient way to increase CREB, or is there something unique about the simultaneous activation of pre- and postsynaptic cells that will encode memory independent of the magnitude of calcium/cAMP/CREB? Recent studies suggest that the simultaneous activation of pre- and post-synaptic neurons leads to a larger influx of calcium into the post-synaptic neuron, which means that there may be nothing mysterious about a Hebbian condition, other than it being a more efficient means for raising $[\mathrm{Ca}]_{i}$ levels, and therefore activating CREB. Such an assumption would imply a direct relation between the simultaneous activation of pre- and post-synaptic activity, calcium, CREB, and memory. 
At the output end, how will an increase in CREB produce a selective change in a specific dendrite that is related to the encoded memory? Several possible mechanisms were proposed recently. Among them is the suggestion that the activated dendrite/synapse is tagged to recruit the newly formed molecules (Frey \& Morris, 1998). Another possibility is based on a recent suggestion that CREB may have a role in the dendritic processing of local synaptic molecules (Crino et al., 1998). These and similar issues are to be addressed in future studies. Such studies will lead us to a better understanding of the formation of new dendritic spines and their relevance to neuronal plasticity.

\section{ACKNOWLEDGEMENT}

This work was supported by a grant from the Binational US-Israel Science Foundation to MS.

\section{REFERENCES}

Bading H, Segal MM, Suche NJ, Dudek H, Lipton SA, Greenberg ME. N-Methyl-D-Aspartate receptors are critical for mediating the effects of glutamate on intracellular calcium concentration and immediate early gene expression in cultured hippocampal neurons. Neuroscience 1995; 64: 653-664.

Bonni A, Ginty DD, Dudek H, Greenberg ME. Serine 133-phosphorylated CREB induces transcription via a cooperative mechanism that may confer specificity to neurotrophin signals. Mol Cell Neurosci 1995; 6: 68183.

Chang F, Greenough WT. Transient and enduring morhological correlates of synaptic activity and efficacy in the rat hippocampal slice. Brain Res 1984; 309: 3536.

Crino P; Khodakhah K; Becker K; Ginsberg S; Hemby S, Eberwine J Presence and phosphorylation of transcription factors in developing dendrites. Proc Natl Acad Sci USA 1998; 95: 2313-2318.

Deisseroth K., Bito H, Tsien, R. Signaling from synapse to nucleus: postsynaptic CREB phosphorylation during multiple forms of hippocampal plasticity. Neuron 1996; 16: 89-101.

Dolmetsch RE, $\mathrm{Xu} \mathrm{K}$, Lewis RS. Calcium oscillations increase the efficiency and specificity of gene expression. Nature 1998; 392: 933-936.

Fifkova E, Van Harreveld A. Long lasting morphological changes in dendritic spines of dentate gcells following stimulation of the entorhinal area. J Neurocytol 1977; 6: $211-230$.

Frey U, Morris RG. Synaptic tagging: implications for late maintenance of hippocampal long-term potentiation. Trends Neurosci 1998; 5: 181-188.

Geinisman Y, Morrell F, deToledo-Morrell, L. Remodeling of synaptic architecture during hippocampal kindling. Proc Natl Acad Sci USA 1988; 85: 32603264 .

Gu G, Rojo AA, Zee MC, Yu J. Simerly RB. Hormonal regulation of CREB phosphorylation in the anteroventral periventricular nucleus. J Neurosci 1996; 16: 3035-3044.

Harris KM, Kater SB. Dendritic spines: cellular specializations imparting both stability and flexibility to synaptic function. Ann Rev Neurosci 1994; 17: 341-371.

Impey S, Mark M, Villacres EC, Poser S, Chavkin C, Storm DR. Induction of CRE-mediated gene expression by stimuli that generate long lasting LTP in area CAl of the hippocampus. Neuron 1996; 16: 973982.

Lamprecht R, Hazvi S, Dudai Y. cAMP response elementbinding protein in the amygdala is required for longbut not short-term conditioned taste aversion memory. J Neurosci 1997; 17: 17-50.

Lee AKS, Schottler F, Oliver M, Lynch G. Brief bursts of high frequency stimulation produce two types of structural change in rat hippocampus. J Neurophysiol 1980; 44: 247-258.

Murphy DD, Segal M. Regulation of dendritic spine density in cultured rat hippocampal neurons is regulated by steroid hormones. J. Neuroscience 1996; 16: 4059-4068.

Murphy DD, Segal M. Morphological plasticity of dendritic spines in central neurons is mediated by activation of CAMP response element binding protein. Proc Natl Acad Sci USA 1997; 94: 1482-1487.

Murphy DD, Cole NB, Greenberger V, Segal M. Estradiol increases dendritic spine density by reducing GABA neurotransmission in hippocampal neurons. J Neurosci 1998; 18: 2550-2559.

Nguyen PV, Kandel ER. A molecular synthesis-dependent late phase of long term potentiation requiring CAMP in the medial perforant pathway of rat hippocampal slices. J. Neurosci 1996; 16: 3189-3198.

Papa M, Bundman MC, Greenberger V, Segal M. Morphological analysis of dendritic spine development in primary cultures of hippocampal neurons. J Neurosci 1995; 15: 1-11.

Papa M, Segal M. Morphological plasticity in dendritic spines of cultured hippocampal neurons. Neuroscience 1996; 71: 1005-1011.

Spaulding SW. The ways in which hormones change cyclic adenosine 3'5'-monophosphate dependent protein kinase subunits, and how such changes affect cell behavior. Endocrine Rev 1993; 14: 632-650. 
Weiland NG, Orikasa C, Hayashi S, McEwen BS. Localization of estrogen receptors in the hippocampus of male and female rats. Society for Neuroscience Abstracts 1996; 22: 618.

Tao X, Finkbeiner S, Arnold DB, Shaywitz AJ, Greenberg ME. $\mathrm{Ca}^{2+}$ influx regulates BDNF transcription factor dependent mechanism. Neuron 1998; 20: 709-720.

Vicario-Abejon C, Collin C, McKay RDG, Segal M. Neurotrophins induce formation of functional excitatory and inhibitory synapses between cultured hippocampal neurons. J. Neuroscience (in press).
Woolley C, McEwen B. Estradiol regulates hippocampal dendritic spine density via an N-methyl-D-aspartate receptor dependent mechanism. J Neurosci 1994; 14: 7680-7687.

Woolley C, Gould E, Frankfurt M, McEwen BS. Naturally occurring fluctuation in dendritic spine density on adult hippocampal pyramidal neurons. J Neurosci 1990; 10: 4035-4039.

Woolley CS, McEwen BS. Estradiol mediates fluctuation in hippocampal synapse density during the estrous cycle in the adult rat. J Neurosci 1992; 12:2549-2554. 

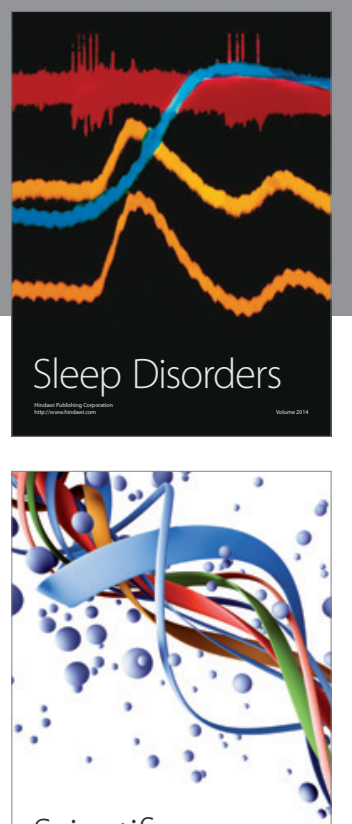

Scientifica
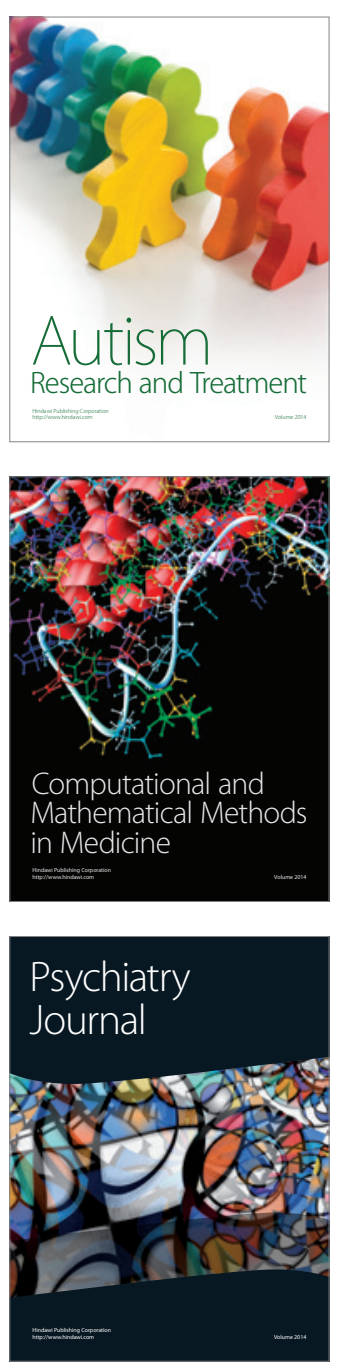
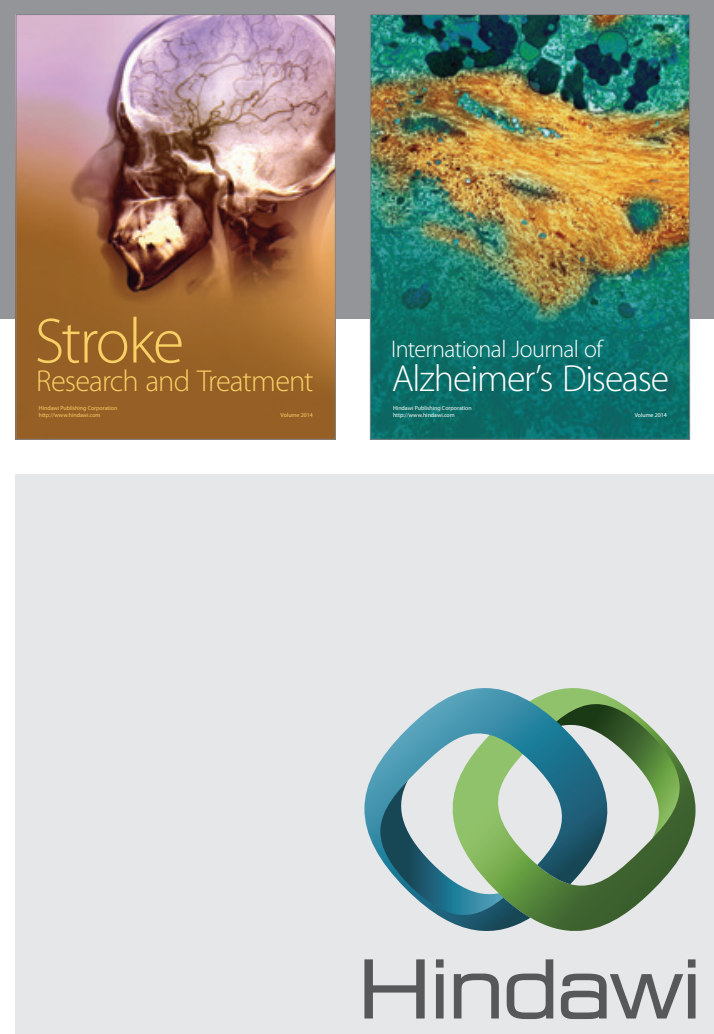

Submit your manuscripts at

http://www.hindawi.com
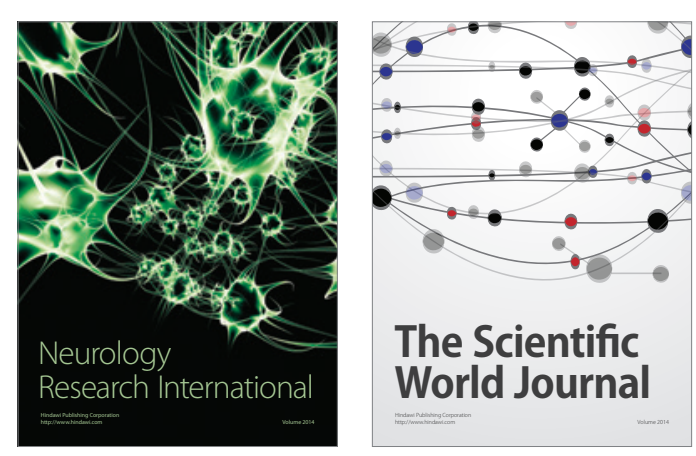

The Scientific World Journal

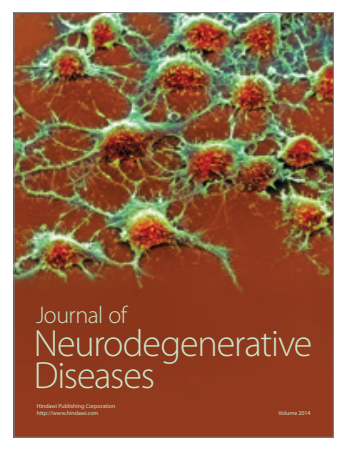

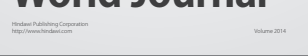

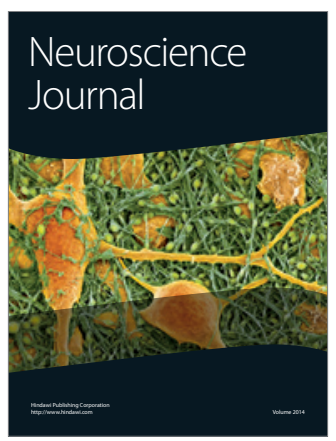

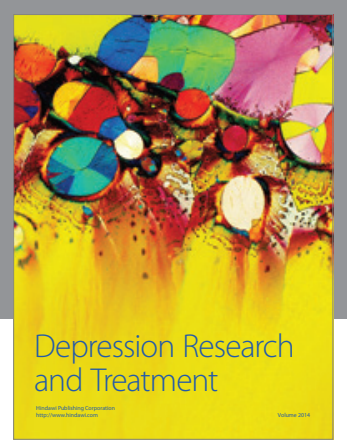
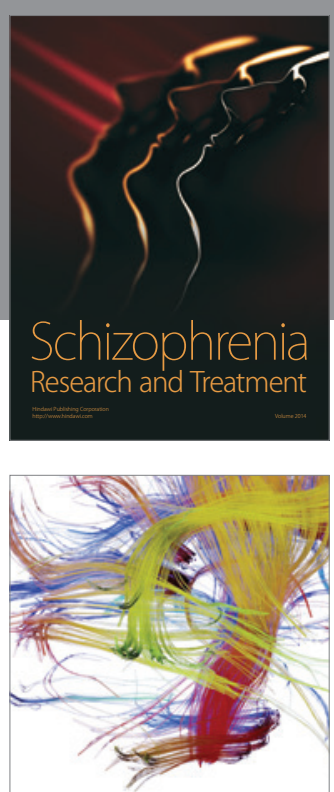

Brain Science

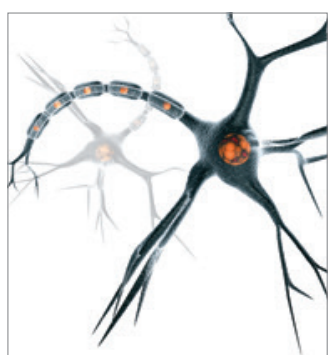

Neural Plasticity
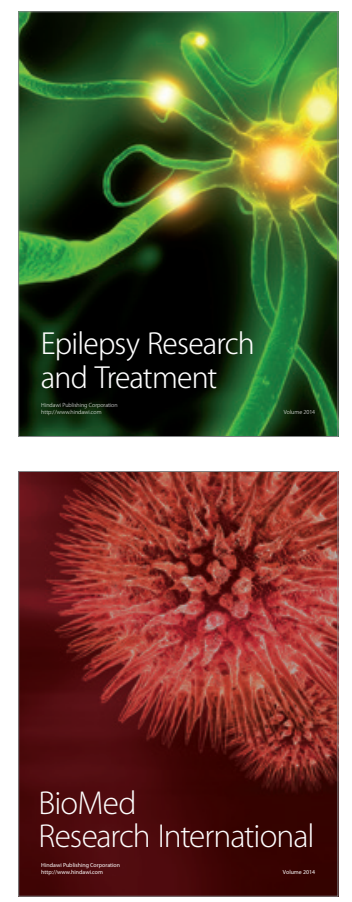

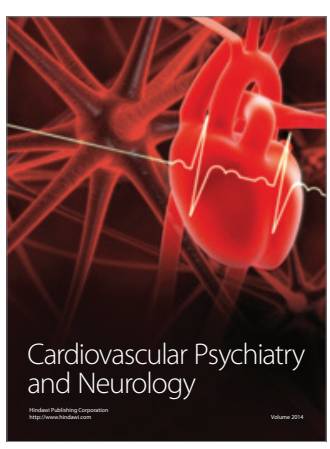

Parkinson's

Disease
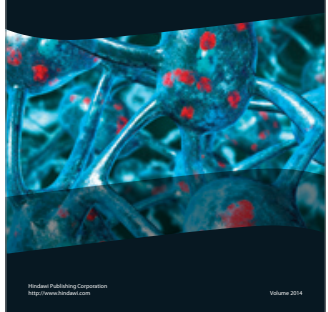